\title{
Effects of collisions on the saturation dynamics of TAEs in tokamaks and stellarators
}

\author{
Christoph Slaby*, Axel Könies, and Ralf Kleiber \\ Max-Planck-Institut für Plasmaphysik, \\ Wendelsteinstraße 1, 17491 Greifswald, Germany \\ José Manuel García-Regaña \\ Laboratorio Nacional de Fusión Ciemat, \\ Av. Complutense 40, 28040 Madrid, Spain
}

(Dated: January 31, 2018)

\begin{abstract}
The non-linear saturation dynamics of TAEs (toroidicity-induced Alfvén eigenmodes) is investigated numerically in tokamaks and stellarators. Special attention is given to the influence that pitch-angle collisions among the fast ions have in the non-linear regime.

For this investigation a perturbative model is used. We employ the three-dimensional ideal reduced MHD eigenvalue code CKA to obtain the mode frequency and mode structure. This information is given to the non-linear gyro-kinetic particle-in-cell code EUTERPE, which calculates the growth rate of the mode and the temporal evolution of the mode amplitude. The mode structure remains fixed for the entire calculation.

In the tokamak, analytical predictions regarding the transition from periodic non-linear behaviour to a steady-state solution and the scaling of the saturated amplitude are available. Both are influenced by collisions. The numerical results are in agreement with the theoretical predictions within the validity range of the theory [H. L. Berk et al., Phys. Rev. Lett. 68, 3563 (1992)]. Beyond the validity range of the theory different scaling laws are found numerically.

We show that using a momentum-conserving collision operator does not change the scaling significantly for small $v$, but is important for high collision frequencies.

The stellarator case, a Wendelstein 7-X high-mirror configuration, shows some differences when compared with the tokamak. Most notably, the saturated perturbed magnetic field becomes a non-monotonic function of $v$.
\end{abstract}

\footnotetext{
* E-mail address: christoph.slaby@ipp.mpg.de
} 


\section{INTRODUCTION}

In fusion devices, present-day experiments or future fusion reactors, there typically exists a supra-thermal population of fast ions. Today, these particles are created mainly by heating methods, such as neutral beam injection (NBI) or ion cyclotron resonance heating (ICRH). In future fusion reactors also self-generated alpha particles (coming from the fusion reaction) will be present. The fast ions are supposed to be confined for a time of the order of the slowing-down time (the time it takes the fast particles to thermalize) in order to heat the plasma. However, the resonant interaction of fast ions with shear Alfvén modes can lead to their destabilization [11, 25] and to increased fastion transport with potentially dangerous consequences for plasma-facing components $[7,35]$. Typical Alfvén eigenmodes excited in tokamaks are toroidicity-induced Alfvén eigenmodes (TAEs), beta-induced Alfvén eigenmodes (BAEs), or - at high fast-particle pressures - energetic-particle modes (EPMs). Because of the more complex geometry of stellarators, other classes of modes, e.g. helicity-induced Alfvén eigenmodes (HAEs), can be destabilized in these devices.

In order to predict transport-induced losses, the non-linear saturation levels of AEs need to be computed. This paper investigates TAEs as an example for the zoo of AEs in general. For tokamaks, it has been shown analytically [2,3] and numerically [20] that the saturation amplitude is influenced by particle collisions. Recently, the influence of pitch-angle collisions on the saturation level of TAEs in NSTX [9] has been investigated [37].

In TJ-II the non-linear dynamics of Alfvén modes, in particular the transition between chirping and steady state, was recently investigated experimentally [22, 23]. It was found that the magnetic configuration plays a larger role for the transition than collisions. Large-scale numerical simulations, aimed at numerically reproducing bursts of Alfvén eigenmodes (AEs) observed in the LHD experiment, have also been performed recently [30]. There, collisions are needed to restore the fast-ion gradient after it has been flattened by an AE burst. They are needed to accurately reproduce experimental measurements.

This paper reports on a systematic numerical study of the non-linear dynamics of TAEs in the saturated phase for various collision frequencies. The simulations are carried out for a tokamak and for a stellarator case. We use a well-established benchmark case devised for tokamaks to compare our numerical results to a theoretically predicted scaling law. The purpose of the tokamak case is two-fold: Firstly, it will serve as a benchmark for our numerical implementation in the parameter range where the analytical theory can be applied. Secondly, the effect of pitch-angle collisions on the saturation dynamics can be studied in other parameter regimes. We find, in particular, that the analytical theory is only applicable in the so-called resonance detuning regime and for low enough collision frequencies.

The numerical model can then be applied to stellarators. As an example, we use the optimized stellarator Wendelstein 7-X (W7-X) - the largest and most sophisticated stellarator in the world [24]. In part, this case is chosen to illustrate the capability of CKAEUTERPE (described below) to also treat real geometries and realistic plasmas. One of the optimization goals of W7-X is good confinement of fast particles, in particular at 
high beta (plasma pressure dived by magnetic pressure). The NBI and ICRH systems are currently being installed at W7-X and will be ready for future experimental campaigns. Thus, now is a natural time to confirm the predictive capabilities of our numerical tools. We use the CKA-EUTERPE code for our numerical simulations. It combines the eigenvalue code CKA (Code for Kinetic Alfvén waves) with the gyro-kinetic particle-in-cell (PIC) code EUTERPE. The coupling of the two codes is perturbative in the sense that an MHD mode is calculated by CKA and then passed to EUTERPE which calculates the motion of fast ions in the pre-calculated field and their power transfer to the mode. Knowing this, the amplitude of the mode can be advanced in time. The mode structure remains fixed throughout the simulation. It is well known that the MHD mode structure may react to the kinetic influence of the fast particles (see e.g. Refs. [28, 34]). Furthermore, the linear mode structure can change in the non-linear phase since it may react to changes of the fast-particle drive [36]. Such effects are not included in the CKA-EUTERPE model. Fully gyro-kinetic simulations, that allow for mode structure variations, would provide a solution to the problem, but suffer from the fact that they are numerically very challenging and expensive. At the moment, they are not suited for parameter scans.

Being a PIC code, EUTERPE is very well suited to compute the collisions between the fast ions. The implementation of collisions into the electromagnetic version of EUTERPE has been benchmarked recently [27]. This reference also serves as a brief introduction to the EUTERPE code. We use a pitch-angle scattering operator acting on the fast-ion distribution function to capture the influence of collisions on the non-linear dynamics (spatial diffusion is neglected). Furthermore, we can choose whether or not the collision operator should conserve momentum. The analytical scaling laws were derived without taking momentum conservation into account. It will be investigated to what extent the inclusion of a momentum-conserving collision operator affects the scaling laws.

This paper is organized as follows. In Sec. II we recapitulate the analytical theory available and briefly introduce the numerical scheme used by CKA-EUTERPE. Sec. III reports on our results for the tokamak case and for the stellarator case, respectively. Finally, conclusions are drawn in Sec. IV.

\section{THEORY AND ALGORITHMS}

To compute the various aspects of (collisional) fast particles interacting with TAEs, the CKA-EUTERPE [10] code package is used. The eigenvalue code CKA [8] computes the real frequency and eigenmode structure of the TAE in the framework of ideal (zero resistivity) magneto-hydrodynamic (MHD) theory. This information is then given to the EUTERPE code which follows numerical marker particles and computes the power transfer from the fast ions to the mode. The wave-particle power transfer is used to calculate a (time-dependent) growth rate $\gamma$, which determines the time evolution of the electromagnetic potentials. Throughout the calculations, the spatial shape of the mode structure remains fixed. Only the complex amplitude is affected by the resonant interaction with the fast particles. In this sense, CKA-EUTERPE is a perturbative model that cannot capture non-linear mode structure modifications. However, this also means that no field equations for the potentials need to be solved, which accounts for the 
higher speed and the enhanced robustness of CKA-EUTERPE compared with fluid or fully gyro-kinetic approaches.

The individual codes are briefly described in the following sections. A more elaborate discussion can be found in Refs. $[8,16]$ and $[15,19]$ for CKA and for EUTERPE, respectively.

\section{A. The CKA code}

CKA (Code for Kinetic Alfvén waves) [8] is a three-dimensional eigenvalue code solving the ideal and reduced MHD equations. It uses a B-spline discretization in all three directions (PEST coordinates), a phase factor to extract the dominant Fourier harmonic, and the electrostatic potential is expressed as $\phi(\mathbf{r}, t)=\phi(\mathbf{r}) \exp (\mathrm{i} \omega t)(\omega$ is the frequency of the mode). The code solves an eigenvalue equation

$$
\omega^{2} D_{2} \phi=D_{1} \phi
$$

where $D_{1}$ and $D_{2}$ are linear differential operators defined as [10]

$$
\begin{aligned}
D_{2} \phi= & \nabla \cdot\left(\frac{1}{v_{\mathrm{A}}^{2}} \nabla_{\perp} \phi\right)+\nabla_{\perp}^{2}\left[\frac{1}{v_{\mathrm{A}}^{2}}\left(\frac{3}{4} \rho_{\mathrm{i}}^{2}+\rho_{\mathrm{s}}^{2}\right) \nabla_{\perp}^{2} \phi\right] \\
D_{1} \phi= & \nabla \cdot\left\{\mathbf{b} \nabla_{\perp}^{2}\left[\left(1-\frac{\mu_{0} p^{(0)}}{B^{2}}\right) \mathbf{b} \cdot \nabla \phi\right]\right\}+\nabla \cdot\left\{\mathbf{b} \times \kappa \frac{2 \mu_{0} \mathbf{b} \times \nabla p^{(0)}}{B^{2}} \cdot \nabla \phi\right\} \\
& -\nabla \cdot\left\{\frac{\mu_{0} j_{\|}^{(0)}}{B}[\nabla \times(\mathbf{b}(\mathbf{b} \cdot \nabla \phi))]_{\perp}\right\} .
\end{aligned}
$$

The notation is conventional: $v_{\mathrm{A}}=B / \sqrt{\mu_{0} n_{\mathrm{i}} m_{\mathrm{i}}}$ denotes the Alfvén speed of the background plasma (characterized by density $n_{\mathrm{i}}$ and ion mass $m_{\mathrm{i}}$ ). $\mathbf{B}$ is the magnetic field with magnitude $B$ and direction $\mathbf{b}$. The subscripts $\|$ and $\perp$ are used to denote vector components parallel and perpendicular to the background magnetic field, respectively. $\mu_{0}$ is the vacuum permeability. $\rho_{\mathrm{i}}=\sqrt{k_{\mathrm{B}} T_{\mathrm{i}} m_{\mathrm{i}}} /\left(q_{\mathrm{i}} B\right)$ and $\rho_{\mathrm{s}}=\sqrt{k_{\mathrm{B}} T_{\mathrm{e}} m_{\mathrm{i}}} /\left(q_{\mathrm{i}} B\right)$ denote the ion gyro-radius and the sound gyro-radius, respectively. $T$ and $k_{\mathrm{B}}$ are the temperature and Boltzmann's constant, respectively. The charge of a bulk-ion is denoted by $q_{\mathrm{i}}$. The equilibrium pressure and current density are labeled as $p^{(0)}$ and $j_{\|}^{(0)}$, respectively. $\boldsymbol{\kappa}=(\nabla \times \mathbf{b}) \times \mathbf{b}$ denotes the curvature of the magnetic field lines.

After Eq. (1) has been solved for $\phi$, the condition $E_{\|}=0$ can be used to easily obtain the parallel component of the vector potential

$$
A_{\|}=\frac{\mathrm{i}}{\omega} \mathbf{b} \cdot \nabla \phi
$$

The solutions $\phi$ and $A_{\|}$together with $\omega$ are then passed to EUTERPE to calculate the growth rate of the mode in the presence of energetic particles. 


\section{B. The EUTERPE code}

EUTERPE, a global non-linear delta-f particle-in-cell code suited for three-dimensional geometries, solves the gyro-kinetic equation

$$
\frac{\partial f_{s}}{\partial t}+\dot{\mathbf{R}} \cdot \nabla f_{s}+\dot{v}_{\|} \frac{\partial f_{s}}{\partial v_{\|}}+\dot{\mu} \frac{\partial f_{s}}{\partial \mu}=\mathcal{C}\left(f_{s}\right)
$$

of a distribution function $f_{s}$ for species $s$. $\mu$ denotes the specific magnetic moment, $\mu=v_{\perp}^{2} /(2 B)$ and $\mathcal{C}\left(f_{s}\right)$ is a collision operator that will be specified later. Eq. (5) is solved together with field equations for the potentials, where particle and current density are calculated by taking moments of the distribution function.

In CKA-EUTERPE, however, typically only one fast-ion species is simulated ( $s=$ fast). Therefore, the species index will be omitted for the remainder of the paper, except where needed to avoid confusion.

The equations of motion (characteristics of Eq. (5)) are solved in the so-called $v_{\|}$ formulation in the absence of collisions:

$$
\begin{aligned}
\dot{\mathbf{R}}= & v_{\|} \mathbf{b}+\frac{m}{q}\left[\frac{\mu B+v_{\|}^{2}}{B B_{\|}^{\star}} \mathbf{b} \times \nabla B+\frac{v_{\|}^{2}}{B B_{\|}^{\star}}(\nabla \times \mathbf{B})_{\perp}\right] \\
& +\frac{v_{\|}}{B B_{\|}^{\star}}\left[\mathbf{b} \times \nabla B+(\nabla \times \mathbf{B})_{\perp}\right]\left\langle A_{\|}\right\rangle+\frac{1}{B_{\|}^{\star}} \mathbf{b} \times \nabla\langle\psi\rangle \\
\dot{v}_{\|}= & -\mu \nabla B \cdot\left[\mathbf{b}+\frac{m}{q} \frac{v_{\|}}{B B_{\|}^{\star}}(\nabla \times \mathbf{B})_{\perp}\right] \\
& -\frac{v_{\|}}{B B_{\|}^{\star}}\left[\mathbf{b} \times \nabla B+(\nabla \times \mathbf{B})_{\perp}\right] \cdot \nabla\langle\phi\rangle \\
& -\frac{\mu}{B_{\|}^{\star}}\left[\mathbf{b} \times \nabla B \cdot \nabla\left\langle A_{\|}\right\rangle+\frac{1}{B} \nabla B \cdot(\nabla \times \mathbf{B})_{\perp}\left\langle A_{\|}\right\rangle\right] \\
\dot{\mu}= & 0
\end{aligned}
$$

with

$$
\begin{aligned}
\psi & =\phi-v_{\|} A_{\|} \\
B_{\|}^{\star} & =B+\left[\frac{m}{q} v_{\|}+\left\langle A_{\|}\right\rangle\right] \mathbf{b} \cdot \nabla \times \mathbf{b} .
\end{aligned}
$$

$\langle\ldots\rangle$ denotes the gyro-average. The ideal Ohm's law

$$
\frac{\partial\left\langle A_{\|}\right\rangle}{\partial t}+\mathbf{b} \cdot \nabla\langle\phi\rangle=0
$$

has been used to eliminate the partial time derivative of the vector potential in the equation for $\dot{v}_{\|}$. Eqs. (6) - (8) are the full equations of motion with all non-linearities 
retained. Note that the structure of the potentials ( $\phi$ and $\left.A_{\|}\right)$is pre-calculated by CKA. Therefore, no field equations need to be solved. Instead, the amplitude equations

$$
\begin{aligned}
\frac{\partial \hat{\phi}(t)}{\partial t} & =\mathrm{i} \omega\left(\hat{A}_{\|}-\hat{\phi}\right)+2\left(\gamma(t)-\gamma_{\mathrm{d}}\right) \hat{\phi} \\
\frac{\partial \hat{A}_{\|}(t)}{\partial t} & =\mathrm{i} \omega\left(\hat{\phi}-\hat{A}_{\|}\right)
\end{aligned}
$$

describe the temporal evolution of the potentials. $\hat{\phi}$ denotes a complex amplitude. The amplitude equations come from taking the time derivative of the quasi-neutrality condition, where the bulk plasma is described using the ideal and reduced MHD equations. The fast ions are treated gyro-kinetically. Furthermore, the ansatz $\phi(\mathbf{r}, t)=$ $\hat{\phi}(t) \phi_{0}(\mathbf{r}) \exp (\mathrm{i} \omega t)$ (and similar for $A_{\|}$and the pressure) has been used. Averaging over the fast oscillation of the mode (with frequency $\omega$ ) yields Eqs. (12) and (13) for the complex amplitudes that evolve on a much longer time scale. The details of the derivation are described in Ref. $[8,16]$. Note that the amplitude equation for $\hat{\phi}$ includes an ad-hoc damping rate $\gamma_{d}$ used to summarize the various damping mechanisms that would be present in a fully gyro-kinetic simulation. The time-dependent growth rate $\gamma(t)=T(t) /(2 W)$ is calculated from the wave-particle power transfer $\left(f^{(1)}\right.$ denotes the perturbed part of the distribution function.)

$$
T(t)=-\int \mathrm{d}^{3} r \int \mathrm{d} \Gamma B_{\|}^{\star}\left[\frac{m}{\mathrm{Ze} B} \mathbf{b} \times\left(v_{\|}^{2} \boldsymbol{\kappa}+\mu \nabla B\right) \cdot\left(\mathrm{Ze}_{\perp} \phi^{*}(\mathbf{r}, t) f^{(1)}\right)\right]
$$

and divided by the wave energy

$$
W=\int \mathrm{d}^{3} r \frac{\rho_{\mathrm{i}+\mathrm{f}}}{B^{2}}\left|\nabla_{\perp} \phi\right|^{2} .
$$

\section{Treatment of collisions}

EUTERPE offers the possibility of including a collision operator on the right-hand side of the kinetic equation (5). Here, we study the influence of pitch-angle collisions on the saturation dynamics. Therefore, the (test-particle) collision operator is given as

$$
\mathcal{C}_{\text {tp }}=\frac{v}{2} \frac{\partial}{\partial \xi}\left(1-\xi^{2}\right) \frac{\partial}{\partial \xi}
$$

with $\xi=v_{\|} / v$ being the pitch-angle variable. This collision operator is obtained by transforming the full Fokker-Planck operator to guiding-center coordinates and neglecting energy and guiding-center diffusion, as illustrated in e.g. Ref. [13]. The collision frequency $v$ is the self-collision frequency of the fast ions defined as [12]

$$
v=v_{0} \frac{\Phi(x)-G(x)}{x^{3}}
$$

where $x=v /\left(\sqrt{2} v_{\text {th }}\right)$ and $v_{\text {th }}=\sqrt{k_{\mathrm{B}} T / m}$. $\Phi$ and $G$ denote the error function and the Chandrasekhar function, respectively. $v_{0}$ is not calculated self-consistently from 
the temperature and density profile of the fast ions, but rather set as a constant. In a typical fusion experiment, the fast particles collide much more frequently with the background plasma than with themselves. We can therefore treat $v_{0}$ as an 'effective' collision frequency that mainly includes collisions with the bulk.

The collisional process itself is easily implemented into a PIC code. After the 'noncollisional' part of the trajectories (the characteristics of the kinetic equation in the absence of a collision operator) are advanced in time using a fourth-order Runge-Kutta method, a Monte-Carlo collision step is performed. EUTERPE uses a scheme that was (first) reported in Refs. [5, 31] (an earlier version was published in Ref. [29]). The scheme employs a (random) rotation of the velocity vector on a spherical shell. This is done by changing the pitch-angle variable of a particle according to

$$
\xi_{\text {out }}=\sin \chi \sin \lambda \sqrt{1-\xi_{\text {in }}^{2}}+\xi_{\text {in }} \cos \chi
$$

Here, $\xi_{\text {in }}$ and $\xi_{\text {out }}$ denote the pitch-angle variable before and after the collision and $\chi=R \sqrt{2 v \Delta t} . \quad \lambda$ is a random number drawn from a uniform distribution between 0 and $2 \pi$, whereas $R$ is drawn from a Gaussian distribution with expectation value 0 and variance 1 [14]. After the collision, the new values for $v_{\|}$and $\mu$ are calculated from $\xi_{\text {out }}$ using the fact that the energy remains unchanged.

A collision operator constructed in such a fashion has the obvious drawback that it does not conserve linear momentum. Also the conservation of particle number and kinetic energy (guaranteed analytically) may be lost numerically due to rounding errors. The reason is that the test-particle term has been retained, but the field-particle term $\mathcal{C}_{\text {fp }}$, describing the reaction of the background particles, was neglected

$$
\mathcal{C}(f)=\mathcal{C}_{\text {tp }}(f)+\mathcal{C}_{\text {fp }}(f) \cong \mathcal{C}_{\text {tp }}(f) .
$$

In EUTERPE a scheme, presented in detail in Refs. [1, 26, 32], that ensures the conservation of particle number, linear momentum, and kinetic energy to machine precision is implemented. The idea is that an appropriate ansatz for the field-particle term can be made such that the contribution of this term cancels exactly the errors made in the conservation laws. The field-particle term is written as

$$
\mathcal{C}_{\mathrm{fp}}\left(f^{(1)}\right)=[N(\mathbf{v}) \mathcal{N}+P(\mathbf{v}) \mathcal{P}+E(\mathbf{v}) \mathcal{E}] F
$$

with coefficients $\mathcal{N}, \mathcal{P}$, and $\mathcal{E}$ that have to be determined. $F$ is the Maxwellian background and

$$
\begin{aligned}
& N(\mathbf{v})=v-3 \sqrt{\frac{\pi}{8}} v_{\mathrm{E}} x^{2} \\
& P(\mathbf{v})=v_{\mathrm{s}} \frac{v_{\|}}{v_{\mathrm{th}}^{2}} \\
& E(\mathbf{v})=v_{\mathrm{E}} x^{2} .
\end{aligned}
$$


For self-collisions, with an externally prescribed collision frequency $v_{0}$, the slowingdown frequency and the energy-diffusion frequency are defined as

$$
\begin{aligned}
& v_{\mathrm{s}}=4 v_{0} \frac{G(x)}{x} \\
& v_{\mathrm{E}}=-2 v+\left(2+\frac{1}{2 x^{2}}\right) v_{\mathrm{s}} .
\end{aligned}
$$

The deflection frequency $v$ is given by Eq. (17).

Writing the field-particle operator in this form, the numerical conservation of the desired quantities can be ensured while keeping the self-adjointness of the collision operator. To determine the coefficients $\mathcal{N}, \mathcal{P}$, and $\mathcal{E}$ the $3 \times 3$ linear system

$$
-\left(\begin{array}{c}
\Delta N \\
\Delta P \\
\Delta E
\end{array}\right)=\int \mathrm{d}^{3} v\left[F\left(\begin{array}{ccc}
N(\mathbf{v}) & P(\mathbf{v}) & E(\mathbf{v}) \\
v_{\|} N(\mathbf{v}) & v_{\|} P(\mathbf{v}) & v_{\|} E(\mathbf{v}) \\
v^{2} N(\mathbf{v}) & v^{2} P(\mathbf{v}) & v^{2} E(\mathbf{v})
\end{array}\right)\right]\left(\begin{array}{c}
\mathcal{N} \\
\mathcal{P} \\
\mathcal{E}
\end{array}\right)
$$

needs to be solved in each spatial bin. The size of one spatial bin is given naturally by the resolution in radial, poloidal, and toroidal direction needed for the representation of the potentials. The quantity $\Delta P$, for example, denotes the 'amount of non-conservation'

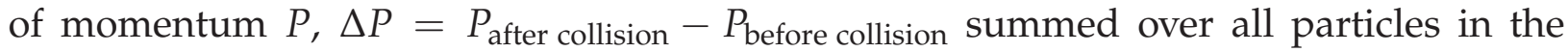
spatial bin (similar for particle number $N$ and kinetic energy $E$ ). Note that in order for the matrix in Eq. (26) to be regular and thus invertible at least two particles are needed in a spatial bin. The scheme can only be applied if this condition is satisfied. Since inverting the $3 \times 3$-matrix is very fast, nearly no increase in runtime is noticeable when the conservation scheme is used.

\section{RESULTS}

Here, we present the results of our non-linear simulations. First, results for a simple circular tokamak are presented. In the second part of this section we show results for a more realistic and more complex W7-X scenario.

\section{A. A tokamak case}

First, the influence of pitch-angle collisions on the saturation dynamics of a TAE in tokamak geometry is presented. We use the well-established ITPA benchmark case [17] for simplicity and because it has become a well-established source for comparison within the fast-particle community. This case therefore provides a link both to recent numerical work [4, 20,33] as well as to analytical theory [2,3], which we also use to benchmark our code. For this test, only pitch-angle collisions of the fast-ions with each other are kept. The collision frequency (meaning $v_{0}$ in Eq. (17)) is considered a parameter and varied over several orders of magnitude (recall that this models also the collisions of fast particles with the background plasma) in order to clearly show all the various nonlinear scenarios that may emerge. The non-linear dynamics and eventual saturation will 
be illustrated based on the temporal behaviour of the perturbed magnetic field of the mode $\delta B$ (more precisely its poloidal component). The value of $\delta B$ at saturation (first maximum) is denoted by $\delta B^{\text {sat }}$.

The magnetic equilibrium is that of a circular, large-aspect-ratio tokamak with concentric circular flux surfaces. The $q$-profile is given by $q(s)=1.71+0.16 s$, with $s$ being the fluxsurface label (normalized toroidal flux). In this geometry we investigate a TAE located near the avoided crossing of the $m=10, n=-6$ and $m=11, n=-6$ continuum branches. The mode amplitude is zero at $s=0$ and $s=1$. The TAE mode is driven unstable by the interaction with a Maxwellian population of fast ions (hydrogen) with a non-uniform density profile given by (see Appendix A for the coefficients)

$$
N_{\text {fast }}(s)=c_{1} \cdot N_{\text {fast }, 0} \exp \left[-\frac{c_{2}}{c_{3}} \tanh \left(\frac{\sqrt{s}-c_{4}}{c_{2}}\right)\right] .
$$

The density gradient is the source of free energy to drive the mode unstable. The temperature of the fast ions is uniform with $T_{\text {fast }}=400 \mathrm{keV}$. Numerical simulations are performed for three different values of the fast-ion peak density

$$
N_{\text {fast }, 0}=\{0.721,1.44,7.21\} \cdot 10^{17} \mathrm{~m}^{-3} \text {. }
$$

Varying the fast-ion density corresponds to changing the linear growth rate $\gamma_{L}$, which is constant for a given case.

Without collisions we compute

$$
\gamma_{\mathrm{L}} \cong\{0.280,1.52,9.53\} \cdot 10^{4} \mathrm{~s}^{-1} \text {. }
$$

Thus, both the resonance detuning and the radial decoupling regime $[4,33]$ are covered (see Fig. 1) in the simulations. It will be investigated if the change of the saturation mechanism $\left(\delta B^{\text {sat }} \propto \gamma_{\mathrm{L}}^{2}\right.$ transitions to $\left.\delta B^{\text {sat }} \propto \gamma_{\mathrm{L}}\right)$ also changes the scaling of the saturated amplitude with respect to $v_{0}$.

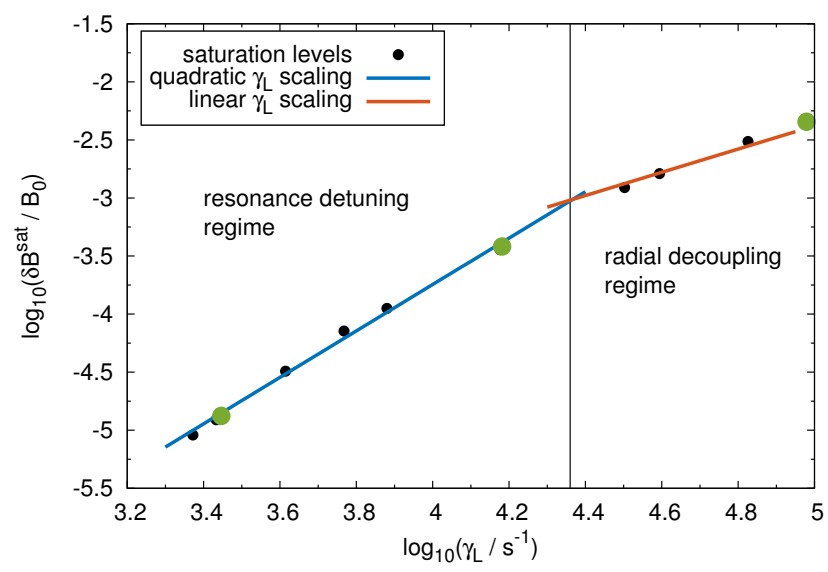

Figure 1: Transition from the resonance detuning regime to the radial decoupling regime without collisions as described in e.g. Refs. [4, 33]. The saturation levels indicated by the black dots have been taken from Ref. [16]. The green dots indicate the cases investigated in the present paper for various collision frequencies. We will refer to them as low-density, medium-density, and high-density case, respectively. Both the resonance detuning and the radial decoupling regime are covered. 
Since the saturation mechanisms are different in the resonance detuning regime versus the radial decoupling regime, it cannot necessarily be expected that collisions will have the same influence on the saturation dynamics in both regimes. Saturation is reached in the resonance detuning regime, because the radial excursions of particles from a flux surface become comparable to the (finite) resonance width where wave-particle power transfer is possible. In the radial decoupling regime, the mode width limits the power transfer. We expect that collisions have a stronger impact on the saturation amplitude in the resonance detuning regime, because this is the regime in which the wave-particle resonance condition is important. The resonance condition includes the parallel velocity of a particle which is directly influenced by collisions. On the other hand, in the radial decoupling regime, saturation is reached because the resonance region becomes wider than the mode-localization region. This is a mechanism that is less sensitive to the individual particles and it should therefore be less influenced by collisions.

Fig. 2 shows the perturbed component of the poloidal magnetic field for various collision frequencies and for the three different fast-ion densities investigated. (The fast-ion density, and therefore also the linear growth rate, increases from top to bottom.) 

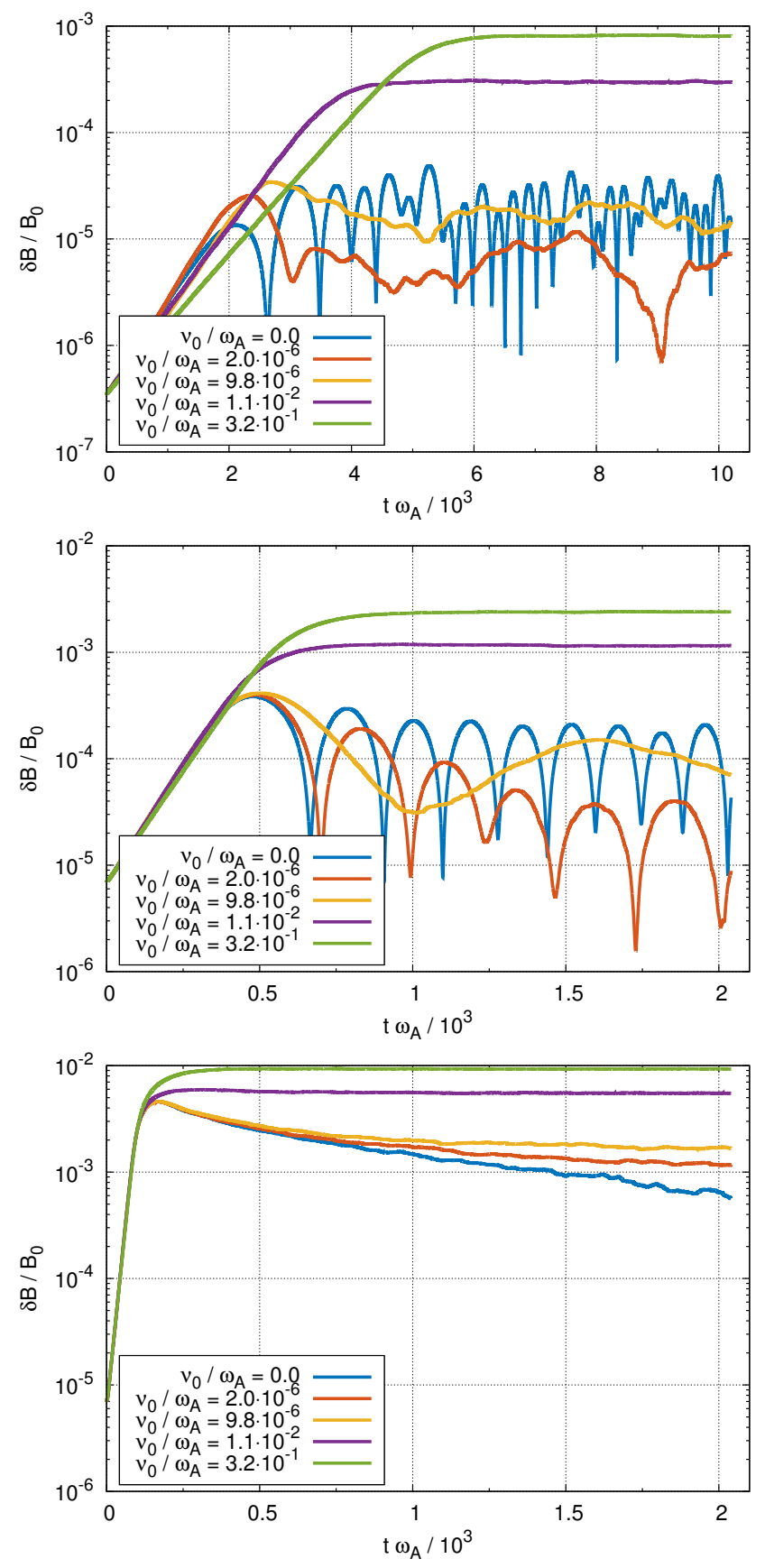

Figure 2: Time trace of the poloidal component of the perturbed magnetic field for the lowdensity (top), medium-density (middle), and high-density case (bottom) for various collision frequencies. Depending on the linear drive and collisionality either a steady-state or a periodic non-linear dynamics develops. The saturation levels (i.e. the first maxima of $\delta B$ ) increase monotonically with $v_{0}$.

All sub-figures clearly show that the non-linear dynamics is influenced by collisions. This includes not only the saturation level itself, but also the dynamics in the saturated phase (saturated phase refers to all times after the first maximum of $\delta B$ ). While the first and the second diagram of Fig. 2 show a similar non-linear dynamics, the high- 
density case on the bottom is different because any periodic non-linear behaviour is completely absent. Between the first two plots and the last one of Fig. 2 the saturation mechanism changes from resonance detuning to radial decoupling. In the high-density case the damping rate (a free input parameter in CKA-EUTERPE, $\gamma_{d}=1.05 \cdot 10^{4} \mathrm{~s}^{-1}$ for all cases) is much smaller than the linear growth rate. Therefore a steady-state solution develops after saturation. It was shown in Ref. [3] that a sufficiently high $\gamma_{\mathrm{d}}$ is required for a periodic scenario to develop.

For the two cases with lower $N_{\text {fast }, 0}$ that show periodic solutions in the non-linear phase, the transition to a more steady-state dynamics happens for a collision frequency $v_{0}$ that is consistent with analytical theory [3]. Note that only for those two cases the analytical theory is valid in the first place. The transition from the periodic regime to the steady-state regime is determined by the relative strength of damping effects

compared with the rate at which the distribution function is rebuilt, $v_{\mathrm{eff}, 0}=v \omega^{2} / \gamma_{\mathrm{L}}^{2}$, [3]. Thus, a good indication of whether a steady-state or a periodic saturation should be expected is to compare $v_{\mathrm{eff}, 0}$ with the damping rate $\gamma_{\mathrm{d}}$. For $v_{\mathrm{eff}, 0}>\gamma_{\mathrm{d}}$ a steady state is expected, whereas the saturation should be periodic for $v_{\text {eff }, 0}<\gamma_{\mathrm{d}}$ [3]. For the ITPA case investigated here, the damping rate equals $v_{\text {eff, } 0}$ for $v_{0} \approx 3.35 \cdot 10^{-7} \omega_{\mathrm{A}}$ (low-density case) and for $v_{0} \approx 9.89 \cdot 10^{-6} \omega_{\mathrm{A}}$ (medium-density case). $\left(\omega_{\mathrm{A}}=v_{\mathrm{A}} / R_{0}\right.$ is the on-axis Alfvén frequency.) Hence, these are the collision frequencies for which the transition between the two regimes should be happening. Re-examining Fig. 2, this is exactly what is found by CKA-EUTERPE. For the low-density case the periodic solution is predicted to disappear for very low collision frequencies. Hence, even for the lowest frequency shown in the plot, $v_{0} / \omega_{\mathrm{A}}=2.0 \cdot 10^{-6}$ (i.e. the red line), the oscillations have already vanished. For the medium-density case (middle plot of Fig. 2), the transition is nicely visible for $v_{0} / \omega_{\mathrm{A}}=9.8 \cdot 10^{-6}$ (see the yellow line) which is close to the theoretically predicted value.

\section{The scaling law}

In order to extract a scaling law, $\delta B^{\text {sat }}\left(v_{0}\right)$, from the simulation data, we take the first maximum in the time-trace of the perturbed magnetic field (Note that, to account for the noisiness of the simulation and to calculate error bars, an average around the presumed location of the maximum is taken.). The analytical theory [3] has been derived for the value of the first maximum. The value $\delta B^{\text {sat }}\left(v_{0}=0\right)$ without collisions is subtracted, and the resulting quantity is plotted as a function of the collision frequency. This has been done in Fig. 3 for the three different fast-ion densities under investigation. 

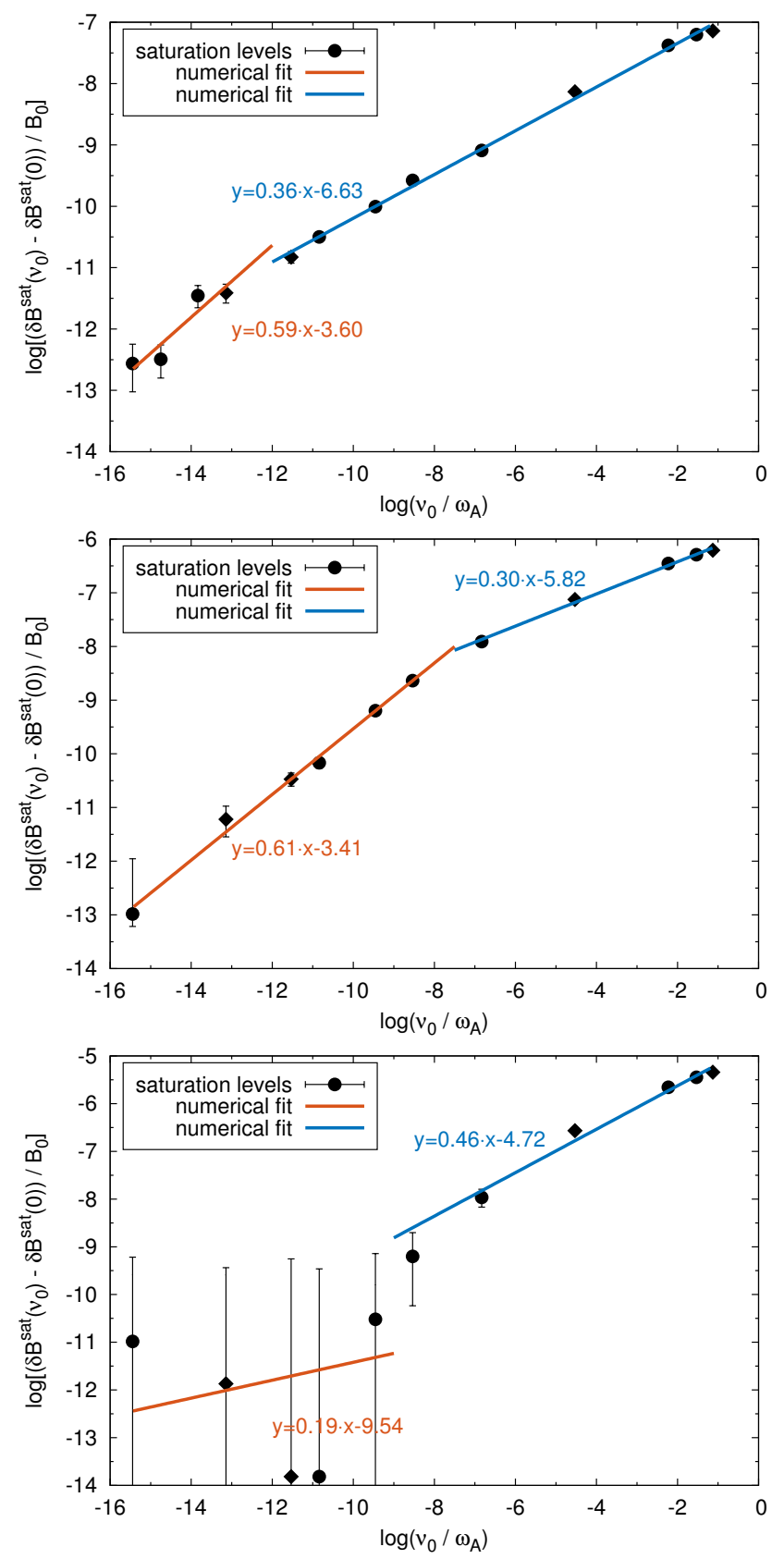

Figure 3: Scaling laws $\delta B^{\text {sat }}\left(v_{0}\right)$ extracted from the simulation data for the low-density (top), medium-density (middle), and high-density case (bottom). The theoretically predicted $v^{2 / 3}$ scaling is confirmed in the resonance detuning regime (top two plots) for low enough collision frequencies (red curves) within the validity range of the theory. For higher collision frequencies different scaling laws are found numerically. The plot on the bottom (radial decoupling regime) shows a characteristic plateau for small $v_{0}$ where the saturation level scales very weakly with collisionality.

The figure show the simulation results (black dots and diamonds) and numerical fits to the data (coloured lines). Note that numerical simulations for more collision frequencies than shown in Fig. 2 have been performed. The cases already presented in Fig. 2 are 
indicated as diamonds in Fig. 3. For all fits a function of the type

$$
y=a \cdot x+b
$$

with $y=\log \left[\left(\delta B^{\text {sat }}\left(v_{0}\right)-\delta B^{\text {sat }}(0)\right) / B_{0}\right]$ and $x=\log \left(v_{0} / \omega_{\mathrm{A}}\right)$ is used. The parameters $a$ and $b$ are determined by the fitting routine. The complete results for all fit parameters (showing also their uncertainties) are given in Appendix B.

In all three cases different scaling laws emerge. The general trend is that small collision frequencies influence the saturation level more strongly if the linear growth rate is small (i.e. if the fast-ion density is small). In the opposite limit, for high growth rates, a 'plateau' forms for small $v_{0}$ indicating that in this regime the saturation level is almost independent of the collision frequency. This fact strengthens our initial assumption that the scaling $\delta B^{\text {sat }}\left(v_{0}\right)$ should be stronger in the resonance detuning regime. Note that if $\delta B^{\text {sat }}\left(v_{0}\right)-\delta B^{\text {sat }}(0)$ became negative, we took the absolute value in order to calculate the logarithm. Furthermore, the error bars are large if $\delta B^{\text {sat }}\left(v_{0}\right)$ and $\delta B^{\text {sat }}(0)$ are close together.

Analytical theory [3] predicts a $v^{2 / 3}$-scaling of the saturated amplitude. Here, the term 'saturated amplitude' is used to refer to the first maximum after the linear phase. This scaling is valid for $\gamma_{\mathrm{L}} \gg \gamma_{\mathrm{d}}, v_{\text {eff }}$. $v_{\text {eff }}$ is the rate of reconstruction of the distribution function after it has been flattened by particle trapping in the wave [3]. Fig. 2 confirmed that, depending on the value of $v_{\mathrm{eff}, 0}$, either a steady state or a periodic scenario emerges. This, however, has no influence on the scaling law, since up until the first maximum of $\delta B$ (corresponds to the first flattening of the distribution function) the physical processes in both regimes are similar and they only differ in the rate of reconstruction of the distribution function after the initial flattening.

Looking at the medium-density case, for which the saturation mechanism is resonance detuning (cf. Fig. 1), the analytically predicted scaling can be confirmed very well for low enough collision frequencies. For large collision frequencies, on the other hand, a different scaling is obvious. We speculate that the condition $\gamma_{\mathrm{L}} \gg v_{\text {eff }}$ is violated before the mode reaches saturation in this cases and, therefore, the theory breaks down. It is intuitively clear that if $\nu_{0}$ is arbitrarily high this is the case. A precise prediction of the collision frequency for which the scaling changes, hinges on a good estimate of $\omega_{\mathrm{b}}$, which is necessary to calculate $v_{\text {eff }}=v \omega^{2} / \omega_{\mathrm{b}}^{2}[3]$.

An instructive estimate for $\omega_{\mathrm{b}}$ can be found in a cylindrical plasma. In the absence of a mode a particle just follows the magnetic field lines. On the other hand, if a mode with a high enough amplitude is present, the resulting $\mathbf{E} \times \mathbf{B}$-drift may significantly influence the particle trajectory. A calculation, detailed in Appendix C, provides the estimate

$$
\omega_{\mathrm{b}}^{2} \cong-\frac{m^{2}}{R_{0}} \frac{v_{\|}}{r B_{0}} \frac{\partial \iota}{\partial r} \phi_{0}
$$

for the bounce frequency of a particle trapped in the wave. ( $\iota=1 / q$ is the rotational transform.) Substituting the parameters of the ITPA benchmark case (medium density) yields a critical collision frequency $v_{\text {crit }} / \omega_{\mathrm{A}} \approx 1.3 \cdot 10^{-4}$ above which the analytical theory can no longer be valid. This critical collision frequency is in the same order of magnitude as the frequency for which the scaling changes in Fig. 3 (middle). Note that 
the simplified analytical model only provides a qualitative estimate for when the scaling law might change. For the low-density case we estimate $v_{\text {crit }} / \omega_{\mathrm{A}} \approx 1.2 \cdot 10^{-6}$.

For the low-density case the estimated value for $v_{\text {crit }}$ is less successful in describing the numerical findings. The trend remains, however, that the region of validity of the analytical theory is much smaller since the linear growth rate is significantly reduced. Therefore, the transition from one scaling law to another happens on the very left of the curve for low collision frequencies. The scaling found numerically for small collision frequencies is still close to the analytical prediction albeit the large uncertainties in the numerical simulations.

Compared with the first two diagrams, the last plot in Fig. 3 is qualitatively different. Since the growth rate is high, the saturation mechanism is radial decoupling and is not described by the analytical theory. Therefore, the estimate for $v_{\text {crit }}$ is not applicable to that case. For these reasons, it is not surprising that the scaling laws found numerically deviate significantly from theoretical predictions. As was shown in Ref. [4], the saturated amplitude of the perturbed magnetic field scales linearly with the growth rate in the radial decoupling regime. It remains to be investigated whether this is the reason why also the scaling law for $\delta B^{\text {sat }}$ changes.

\section{The influence of the conservation scheme}

So far, all results presented in this section were obtained without the use of the parallelmomentum conservation scheme. Such a scheme is also absent in Refs. [3, 20,37]. With the predicted $v^{2 / 3}$-scaling confirmed for the medium-density case, it is worthwhile to investigate how the non-linear dynamics and the scaling law change when the conservation of momentum is properly taken into account. Fig. 4 shows the scaling of the saturated amplitudes for the same collision frequencies investigated before for the medium-density case.

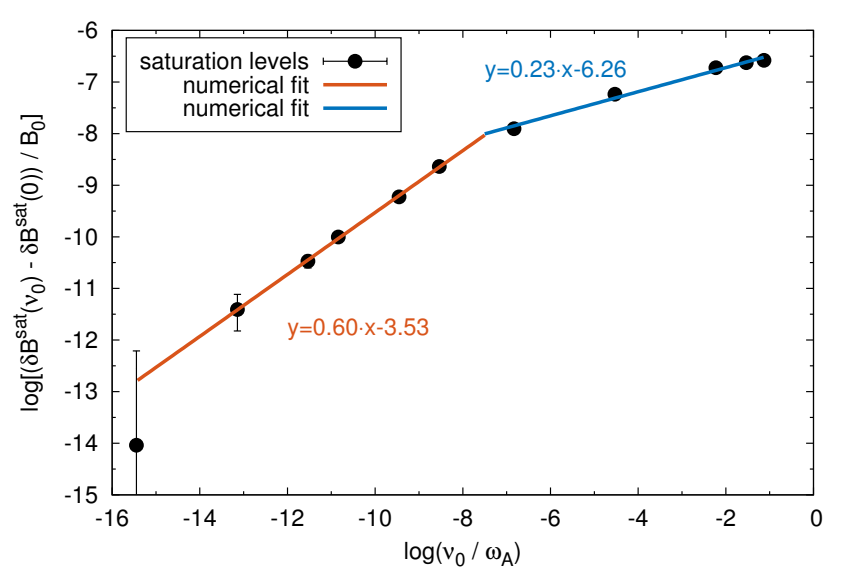

Figure 4: Same as Fig. 3 (middle plot), but taking into account also momentum conservation for the collision operator. Again, two regions with different scaling laws are observed. Momentum conservation is mainly important for the large collision frequencies. The lowest collision frequency was excluded from the numerical fit. 
For small values of $v_{0}$, the saturated amplitudes are very similar with and without the conservation scheme. Consequently, also the scaling is only influenced slightly. On the other hand, for large $v_{0}$ the conservation of momentum by the collision operator becomes important. The scaling changes in the sense that if the momentum correction is included in the collision operator, the saturation levels depend less on $v_{0}$. Neglecting the momentum correction leads to an overestimation of collisional effects just as it does in other areas of tokamak physics, such as neoclassical transport and micro-instability theory [14]. We find that conservation of momentum only changes the saturation levels, but does not affect the non-linear dynamics. For example, the transition from the periodic non-linear behaviour to the steady-state solution remains unchanged.

Note that the conservation scheme is designed for self-collisions. Even though $v_{0}$ contains fast-fast collisions as well as fast-ion collisions, no momentum is transferred to the background ions.

\section{Saturation mechanism - density flattening}

All the numerical simulations share the feature that the mode amplitude saturates because the density profile of the fast ions flattens in the region of interest. The cases with non-vanishing collision frequency exhibit a stronger flattening of the density profile. We attribute this only to a 'secondary' effect of collisions. As has been shown, collisions lead to a higher saturation level, i.e. a larger mode amplitude, which in turn leads to enhanced fast-particle transport.

The high-density case with the highest $v_{0}$-value is taken as an example in Fig. 5, since this case has the highest saturation level of all cases investigated.

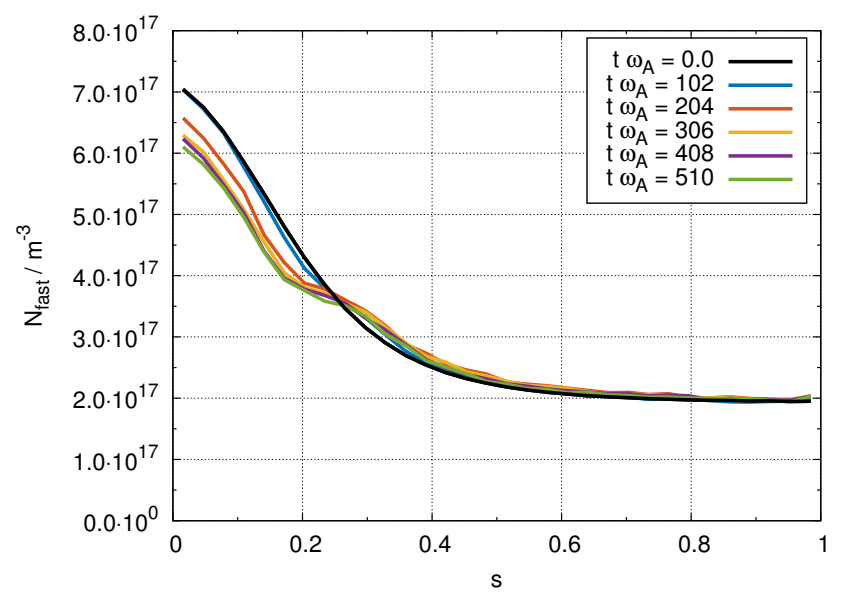

Figure 5: Flattening of the fast-ion density profile at various times in the simulation for the high-density case with the highest collision frequency. Since the saturation mechanism is radial decoupling flattening of the profile occurs in broad region comparable to the mode width. The flattening is significant because the saturation level is enhanced due to collisions.

Flattening of the density profile over the region where the mode is localized (characteristic for the radial decoupling regime) is very obvious for later times in the simulation. 
Note that due to numerical inaccuracies, about $4 \%$ of the fast-ion density is lost over the course of the simulation.

For the medium-density case without collisions the initial profile and the saturated profile at the end of the simulation (in the time-asymptotic limit) are compared in Fig. 6 on the left-hand side.
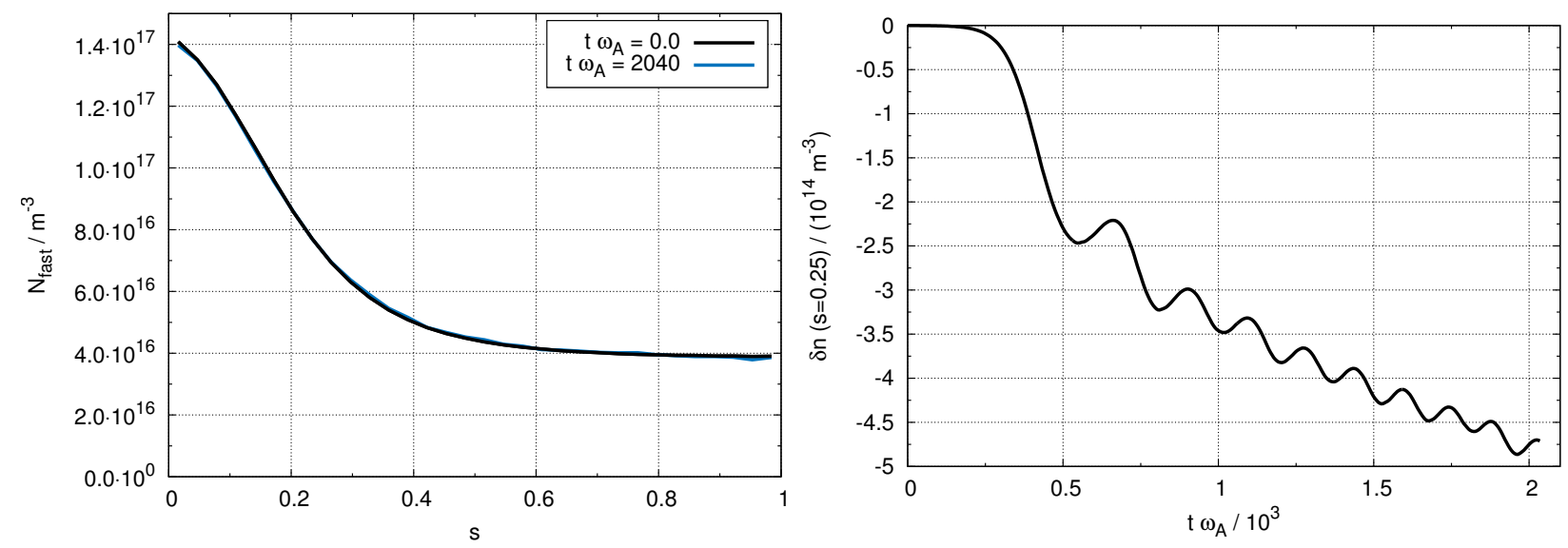

Figure 6: Left: Flattening of the fast-ion density profile for the medium-density case without collisions at a late time in the simulation. Since the saturation mechanism is resonance detuning flattening of the profile occurs in a narrow region.

Right: Instantaneous fast-ion density at $s=0.25$. Overall, particles are transported away from the mode-localization region. The particle density oscillates in time with the maxima and minima being correlated to the amplitude of the perturbed magnetic field as shown in Fig. 2 in the middle.

Since the saturation level is low, transport is low and no significant changes can be seen in the saturated density profile. Note that in the resonance detuning regime the flattening of the density profile can be restricted to a very narrow region, which is challenging to resolve numerically. It is, however, possible to investigate the instantaneous change in the fast-ion density profile, as shown in Fig. 6 on the right-hand side, caused by the presence of the TAE. The change in particle density is investigated at the radial position $s=0.25$ where the mode is peaked. Clearly, particles are transported away from the mode-localization region. By the end of the simulation, the particle density at $s=0.25$ has decreased by approximately $0.6 \%$. This is why the change is not visible by looking directly at the profile. The fast-ion density is oscillating in time in such a way that the fast-ion density is minimal (local minima in time) whenever the mode amplitude is largest (cf. the middle plot of Fig. 2). This corresponds to a flattening of the density gradient, followed by a phase of mode decay. In this phase, the density gradient can recover and the whole process starts anew.

\section{B. Convergence scan}

A convergence test with respect to time step and marker number has been performed for the medium-density case at the highest collision frequency. Fig. 7 shows the results 
in terms of the perturbed magnetic field as calculated by the code. Both diagrams (time step halved on the left-hand side and numerical marker number doubled on the righthand side) confirm that the standard resolution was chosen well. The standard time step for all tokamak calculations is $\Delta t \omega_{\mathrm{A}}=0.1$. We used $N_{\mathrm{p}}=10^{6}$ numerical marker particles in the standard runs.
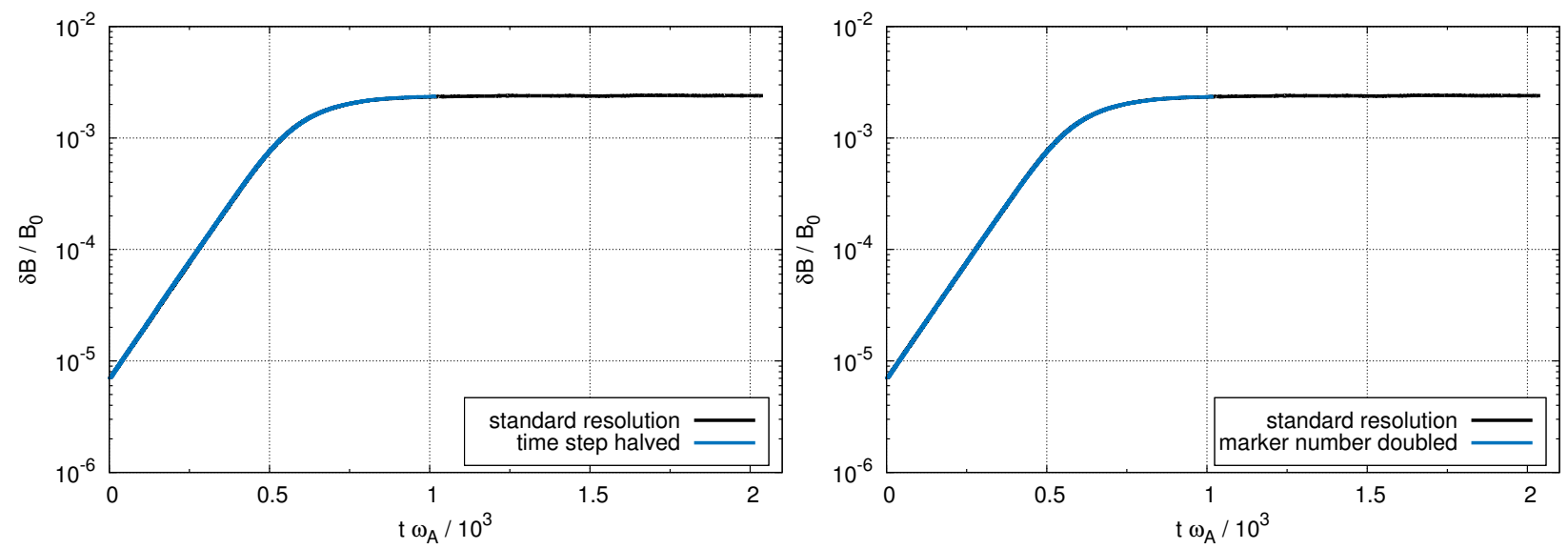

Figure 7: Convergence scan with respect to time step (left) and maker number (right). Since the deviations between the curves are below $1 \%$ (measuring $\gamma_{\mathrm{L}}$ ), the standard resolution was chosen well.

It has been reported before that for the particular collisional scheme used in EUTERPE the time step is more critical than the number of markers [27]. Nevertheless, the relative deviations in the linear growth rate (compared with the standard resolution) are well below $1 \%$, which is very acceptable.

\section{A stellarator case}

Having confirmed the theoretically predicted scaling law in tokamaks (in the resonance detuning regime and for low enough collision frequencies), we now apply the CKAEUTERPE code to a Wendelstein 7-X (W7-X) high-mirror equilibrium with an on-axis magnetic field $B_{0}=2.44 \mathrm{~T}$. This confirms that CKA-EUTERPE can handle realistic geometries and plasmas. Due to favourable properties regarding the confinement, the so-called high-mirror case (characterized by a large $B_{1,-1}$ Fourier component of the magnetic field) has become standard for performing numerical simulations. It is characterized by an $\iota$-profile close to unity as can be seen in Fig. 8, which also shows the density profile of the fast ions used in the numerical simulations. The on-axis fast-ion density is $N_{\text {fast }, 0}=5 \cdot 10^{18} \mathrm{~m}^{-3}$. The fast-particle temperature profile is flat with a value $T_{\text {fast }}=55 \mathrm{keV}$. Since stellarators are numerically more challenging than tokamaks, the time step is chosen as $\Delta t \omega_{\mathrm{A}}=0.08$ and marker number is increased to $N_{\mathrm{p}}=5 \cdot 10^{6}$. 


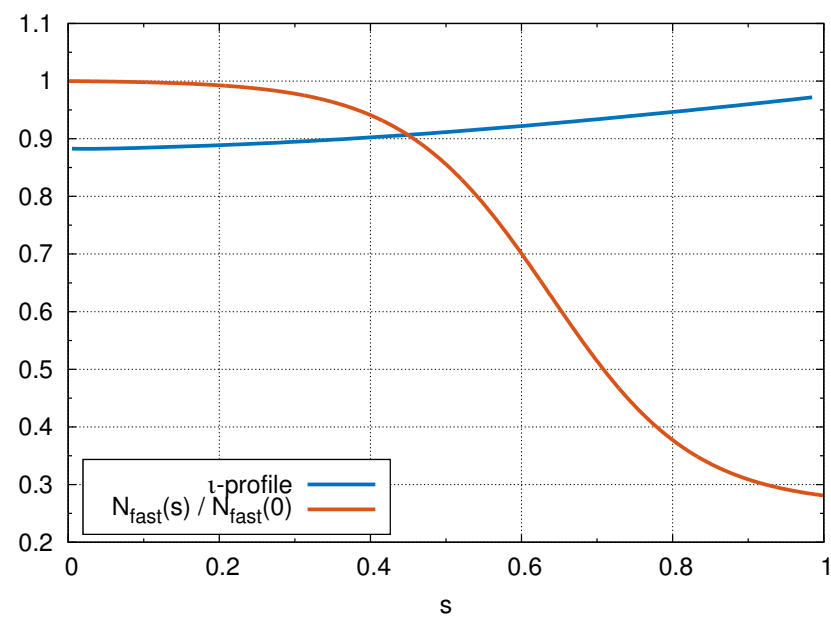

Figure 8: $\iota$-profile and fast-ion density profile (normalized to on-axis value) for the Wendelstein 7-X (W7-X) high-mirror configuration.

The continuous Alfvén spectrum (neglecting sound modes) as well as the MHD mode structure are shown in Fig. 9.
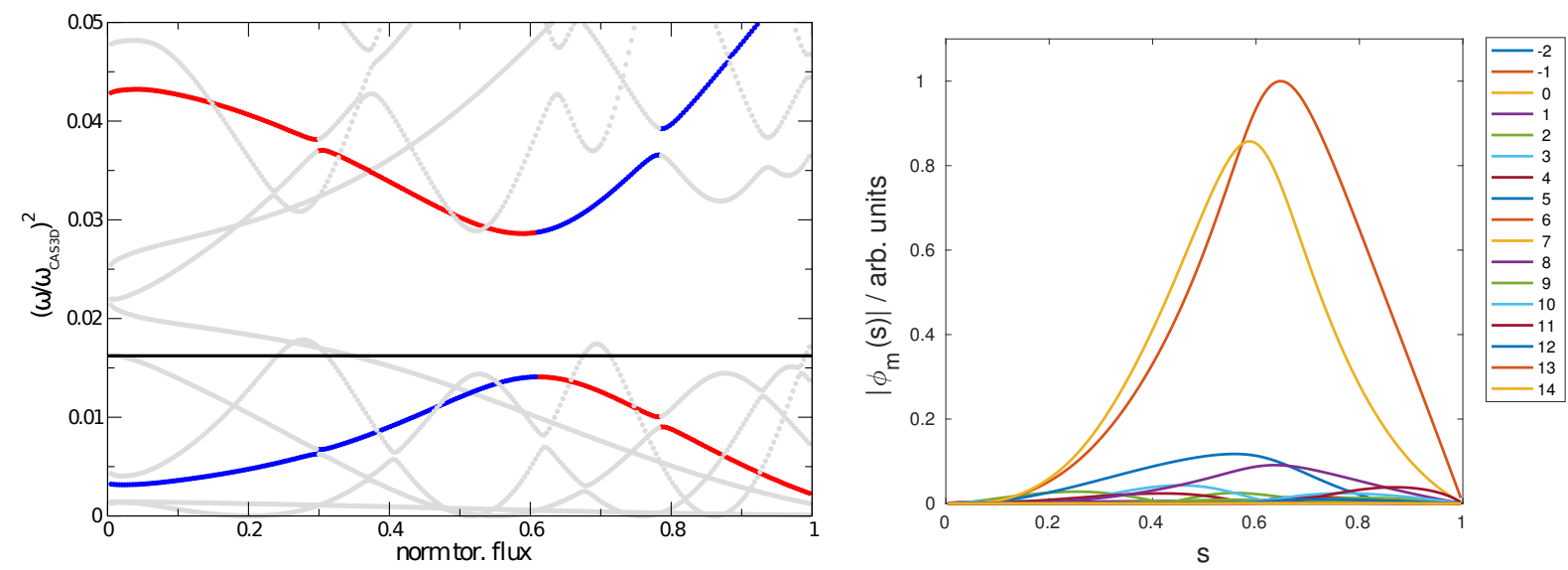

Figure 9: Left: Shear Alfvén wave continuum for the W7-X high-mirror configuration. The relevant continuum branches $(6,-6$, red) and $(7,-6$, blue) are coloured. The TAE frequency is indicated as a solid black line.

Right: Radial TAE mode structure (electrostatic potential) as calculated by CKA. The mode extends over a broad radial region.

In the continuum, which was calculated by the CONTI code [18] for a flat bulk-ion density profile $\left(N_{\text {bulk }}=10^{20} \mathrm{~m}^{-3}\right)$, only the relevant continuum branches $(m=6, n=-6$ and $m=7, n=-6$ ) are coloured. The TAE (frequency indicated by a black horizontal line) resides in the TAE gap.

The real frequency and linear growth rate of the mode in the absence of collisions are $\omega=3.36 \cdot 10^{5} \mathrm{~s}^{-1}$ and $\gamma_{\mathrm{L}}=1.76 \cdot 10^{4} \mathrm{~s}^{-1}$, respectively. We now increase the collision frequency as we did before for the ITPA case and investigate the non-linear dynamics of the mode in stellarator geometry. The results are presented in Fig. 10. 

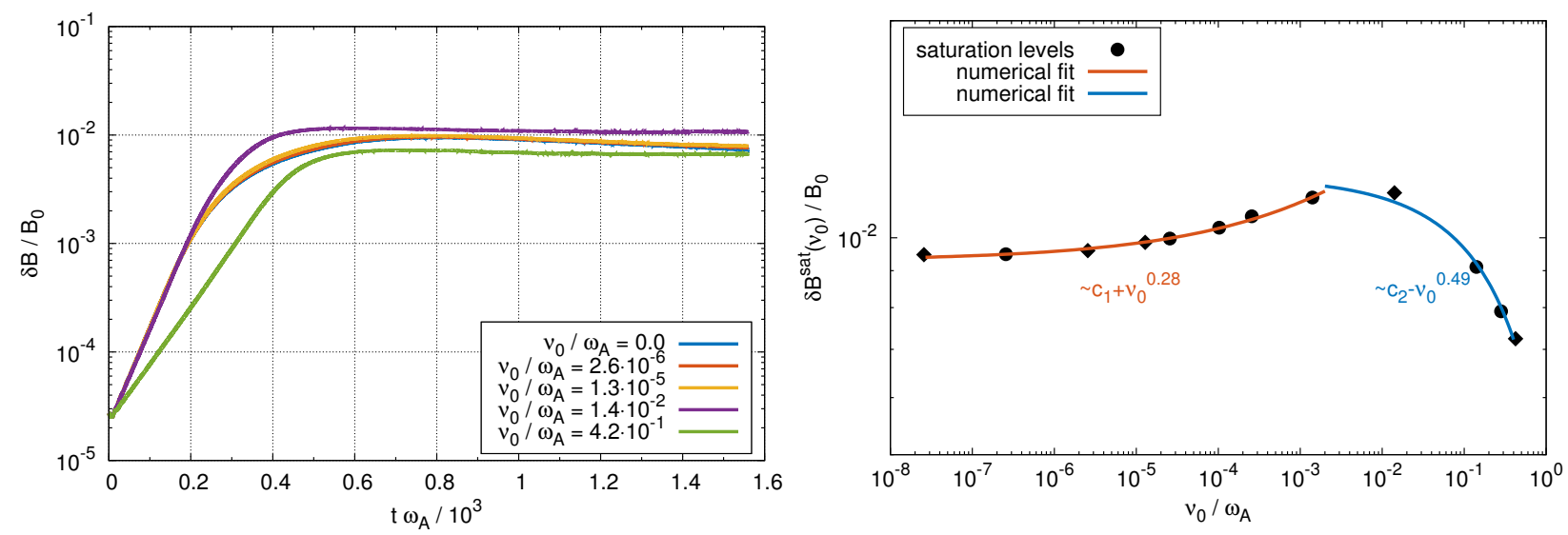

Figure 10: Left: Time trace of the poloidal component of the perturbed magnetic field for the W7-X case. Simulations are performed for various collision frequencies. Any periodic non-linear solutions are absent. The linear growth rate is influenced for high $v_{0}$. Unlike in the tokamak case, the saturation level is a non-monotonic function of $v_{0}$. Note that the very high fast-ion density accounts for the unrealistically large saturation amplitude.

Right: Scaling law $\delta B^{\text {sat }}\left(v_{0}\right)$ extracted from the simulation data. For low collision frequencies the saturation level only scales weakly with $v_{0}$ which could mean that the saturation mechanism is radial decoupling. Note that $c_{1}$ and $c_{2}$ are parameters of the fit (see Tab. $\mathrm{V}$ in the Appendix).

The left-hand side of the figure shows the time trace of the poloidal component of the perturbed magnetic field for a few selected collision frequencies. It can be observed that any periodic behaviour in the non-linear phase is completely absent. (Note that the medium-density tokamak case, which showed periodic behaviour, has a similar growth rate as the stellarator case.) The reason could be that the external damping rate is only $\gamma_{\mathrm{d}}=1.05 \cdot 10^{3} \mathrm{~s}^{-1}$ and that therefore the case is too far away from marginality. We speculate that the saturation mechanism is radial decoupling. This assessment is also supported by the fact that the flattening of the density profile happens over an area of large radial extent comparable to the mode width, see Fig. 11. Shown is the case without collisions. Including collisions makes no qualitative difference. 


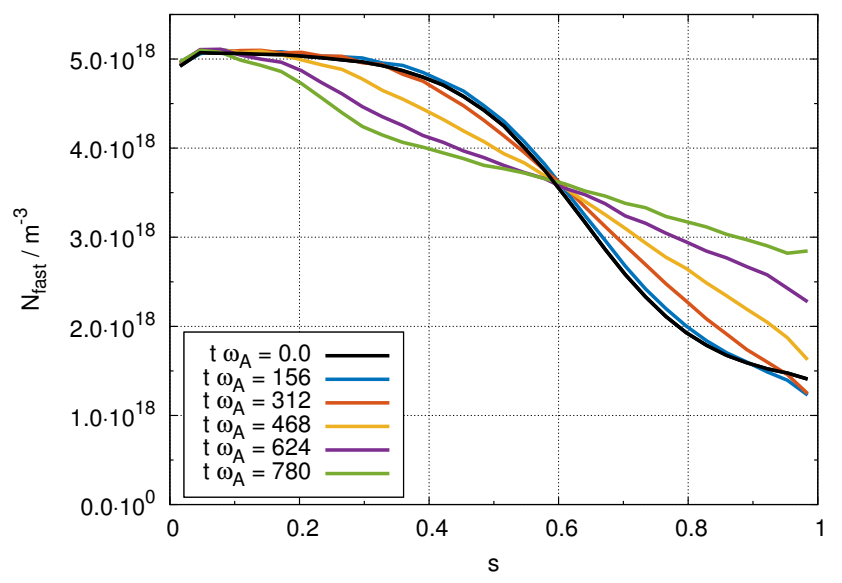

Figure 11: Flattening of the fast-ion density profile at various times in the simulation for the W7$\mathrm{X}$ high-mirror case without collisions. The saturation mechanism resembles radial decoupling and a flattening of the profile over the whole mode-localization region is observed.

About $5 \%$ of the fast-ion density is lost due to numerical errors.

Since the $\iota$-profile of this specific W7-X configuration is very flat (low shear), there is very little variation in $\omega_{\text {res }}$ (as defined in Ref. [4]). Thus, even for very small growth rates, the resonance width can be of the same order as the mode width which then leads to radial decoupling.

It was found in the tokamak case that if the saturation mechanism is radial decoupling, the saturation level scales very weakly with collisionality for low values of $v_{0}$. Examining the right-hand side of Fig. 10, a similar observation can be made for W7-X. However, a striking difference between tokamak and stellarator is that $\delta B^{\text {sat }}$ is a non-monotonic function of $v_{0}$. Large collision frequencies clearly lead to a reduction of the saturation level. It even drops below the the collision-less value.

Unlike in the tokamak cases, the linear growth rate is reduced substantially by collisions (reduction of approx. $42 \%$ for the highest collision frequency compared with the collision-less case). In the tokamak cases, $\gamma_{\mathrm{L}}$ only changes by $20 \%, 7 \%$, and $3 \%$ for the low-density, medium-density, and high-density cases, respectively.

The saturated amplitude of the magnetic field is a function of many parameters with $\gamma_{d}$, $\gamma_{\mathrm{L}}$, and $v_{0}$ being the most important ones. Disentangling the various contributions of the individual parameters to the overall scaling law proved to be successful for the ITPA case, since $\gamma_{d}$ is an external parameter and $\gamma_{L}$ was found to not depend significantly on $v_{0}$.

In the stellarator, $\gamma_{\mathrm{L}}$ and $v_{0}$ cannot be varied independently (at least for high collision frequencies). This may be the reason why $\delta B^{\text {sat }}$ is a non-monotonic function of $v_{0}$. This issue remains to be investigated.

\section{SUMMARY AND CONCLUSIONS}

We have studied the non-linear saturation of TAEs in tokamaks and stellarators. Special emphasis is given to the role of pitch-angle collisions of the fast ions and how the 
collisions influence the saturation level and the subsequent non-linear dynamics in the saturated phase. As a tokamak representative, the ITPA benchmark case was chosen. We performed numerical simulations for the standard case as well as with half of the standard density and a five-times increased value. In doing so, we cover both the resonance detuning and the radial decoupling regime by the simulations.

It is seen that features predicted by analytical theory are recovered by the simulations, if they are performed in the validity range of the theory. We showed that the transition from a periodic dynamics in the non-linear phase to a steady-state solution occurs for a collision frequency that is consistent with analytical predictions. In the resonance detuning regime (i.e. for cases that are closer to marginal stability) the theoretically predicted $v^{2 / 3}$-scaling of the saturation level is confirmed numerically. In this sense, this case also serves as a benchmark of the implementation of collisions into the non-linear and electromagnetic version of CKA-EUTERPE.

Outside the validity range of the theory (for $\gamma_{\mathrm{L}} \lesssim v_{\text {eff }}$ ) scaling laws deviating from the $v^{2 / 3}$-prediction are found numerically. The critical collision frequency $v_{\text {crit }}$ for which the scaling is supposed to change can (roughly) be estimated by calculating the bounce frequency $\omega_{\mathrm{b}}$ of particles trapped in the potential of the wave. A simple cylindrical calculation for $\omega_{\mathrm{b}}$ leads to an estimate for $v_{\text {crit }}$ that qualitatively matches the numerical findings.

Analytical theory and previous numerical simulations did not include a momentumconserving collision operator. We investigated the implications that conserving linear momentum in the collision step has on the scaling law. It turns out that neglecting momentum conservation leads to an overestimation of collisional effects. However, this only becomes important for large collision frequencies. As expected, for small values of $v_{0}$ the scaling laws found numerically with and without the momentum-conservation scheme are the same.

After having confirmed the analytical predictions in tokamaks, the code was applied to a Wendelstein 7-X (W7-X) high-mirror equilibrium. Similarly to the ITPA case a TAE, now with a broader radial structure, is investigated in stellarator geometry. Several differences become apparent when comparing the tokamak and the stellarator case. The stellarator case shows no periodic non-linear behaviour, which might be a consequence of the relative values of $\gamma_{L}$ and $\gamma_{d}$ used for this simulation. The second difference is that the scaling of the saturation level with $v_{0}$ is now non-monotonic. Again, this might be related to a closer entanglement of the various factors influencing the non-linear dynamics in stellarators. The weak scaling of $\delta B^{\text {sat }}\left(v_{0}\right)$ coupled with a flattening of the fast-ion density profile over the whole mode-localization region suggests that the saturation regime in W7-X is radial decoupling. Notwithstanding the above, the mode is found to saturate due to profile flattening in the tokamak and stellarator alike.

Finally, it must be pointed out that it is too soon to draw any final conclusions about the non-linear dynamics of shear Alfvén modes in W7-X. Many more gap modes existing in stellarator geometry (HAEs, EAEs, MAEs, but also EPMs) with different mode numbers and radial profiles need to be studied to determine if the findings reported here prevail in general. Especially with neutral beam injection becoming available at W7-X for the next operation phase, also the effect of using a slowing-down distribution function for the fast-ions and a proper slowing-down collision operator should be studied. The 
implementation of proper distribution functions for such scenarios is in progress.

All numerical simulations reported in this paper were performed in a perturbative sense with the mode structure held fixed throughout the simulations. Future investigations with fully gyro-kinetic models (allowing for the evolution of the mode structure) should be carried out in order to confirm the results of the perturbative models. Such simulations are, however, still very expensive and numerically challenging and the moment not suited for parameter studies as the one reported in this paper.

Finite $\mathbf{A}_{\perp}$-effects have been neglected in this paper. While CKA as a reduced MHD code contains $\mathbf{A}_{\perp}$, the equations of motion in EUTERPE do not. A more complete coupling of CKA and EUTERPE in this regard is a matter of ongoing research.

As has been pointed out in $[6,21]$, when compared to models or numerical predictions, experimentally diagnosed properties of chirping solutions can provide information on plasma parameters which are otherwise difficult to diagnose. Hence, a next step should be to investigate stellarator cases that are closer to marginality and thus allow for chirping behaviour.

\section{Acknowledgment}

The simulations were performed on the MARCONI supercomputer (CINECA), the HPC extension DRACO (MPCDF), and the local Linux cluster in Greifswald.

Christoph Slaby would like to thank Matthias Borchardt and Henry Leyh for their assistance with the parallel computing environment.

This work has been carried out within the framework of the EUROfusion Consortium and has received funding from the Euratom research and training programme 20142018 under grant agreement No 633053. The views and opinions expressed herein do not necessarily reflect those of the European Commission.

\section{Appendix A: Parameters of the ITPA density profile}

The coefficients of the density profile are listed in Tab. I.

Table I: Parameters of the ITPA fast-ion density profile

\begin{tabular}{c|c|c|c|c}
\hline \hline coefficient & $c_{1}$ & $c_{2}$ & $c_{3}$ & $c_{4}$ \\
\hline value & 0.521298 & 0.198739 & 0.298228 & 0.49123 \\
\hline \hline
\end{tabular}

\section{Appendix B: Parameters of the numerical fits}

Tabs. II-IV list all resulting parameters of the numerical fits shown in Fig. 3 in Sec. III. For all fits a function of the type

$$
y=a \cdot x+b
$$

with $y=\log \left[\left(\delta B^{\text {sat }}\left(v_{0}\right)-\delta B^{\text {sat }}(0)\right) / B_{0}\right]$ and $x=\log \left(v_{0} / \omega_{\mathrm{A}}\right)$ is used. 
Table II: Parameters of the numerical fits for the low-density case

\begin{tabular}{c|c|c}
\hline \hline & $a$ & $b$ \\
\hline small $v_{0}$ (red curve) & $0.59 \pm 0.15$ & $-3.60 \pm 2.20$ \\
\hline large $v_{0}$ (blue curve) & $0.36 \pm 0.01$ & $-6.63 \pm 0.05$ \\
\hline \hline
\end{tabular}

Table III: Parameters of the numerical fits for the medium-density case

\begin{tabular}{c|c|c|c|c}
\hline \hline & \multicolumn{2}{|c|}{ without conservation scheme } & \multicolumn{2}{|c}{ with conservation scheme } \\
\hline & $a$ & $b$ & $a$ & $b$ \\
\hline small $v_{0}$ (red curve) & $0.61 \pm 0.03$ & $-3.41 \pm 0.30$ & $0.60 \pm 0.01$ & $-3.53 \pm 0.08$ \\
\hline large $v_{0}$ (blue curve) & $0.30 \pm 0.01$ & $-5.82 \pm 0.04$ & $0.23 \pm 0.02$ & $-6.26 \pm 0.06$ \\
\hline \hline
\end{tabular}

Table IV: Parameters of the numerical fits for the high-density case

\begin{tabular}{c|c|c}
\hline \hline & $a$ & $b$ \\
\hline small $v_{0}$ (red curve) & $0.19 \pm 0.36$ & $-9.54 \pm 4.17$ \\
\hline large $v_{0}$ (blue curve) & $0.46 \pm 0.03$ & $-4.72 \pm 0.14$ \\
\hline \hline
\end{tabular}

In the case of W7-X (see Fig. 10) the data are fitted to

$$
y=b \cdot x^{a}+c
$$

with $y=\delta B^{\text {sat }}\left(v_{0}\right) / B_{0}$ and $x=v_{0} / \omega_{\mathrm{A}}$. Tab. $\mathrm{V}$ lists the results.

Table V: Parameters of the numerical fits for the W7-X case

\begin{tabular}{c|c|c|c}
\hline \hline & $a$ & $b$ & $c$ \\
\hline small $v_{0}$ (red curve) & $0.28 \pm 0.03$ & $0.013 \pm 0.002$ & $0.009 \pm 7.7 \cdot 10^{-5}$ \\
\hline large $v_{0}$ (blue curve) & $0.49 \pm 0.16$ & $-0.008 \pm 8.0 \cdot 10^{-4}$ & $0.012 \pm 6.0 \cdot 10^{-4}$ \\
\hline \hline
\end{tabular}




\section{Appendix C: An analytical estimate for $\omega_{\mathrm{b}}$}

Consider a particle moving in a cylindrical plasma. The magnetic field is given by

$$
\mathbf{B}=B_{1} \mathbf{e}_{\Theta}+B_{0} \mathbf{e}_{z}
$$

where $\mathbf{e}_{\Theta}$ and $\mathbf{e}_{z}$ are unit vectors in cylindrical coordinates. The equations of motion are

$$
\begin{aligned}
\dot{\mathbf{R}} & =v_{\|} \mathbf{b}+\frac{\mathbf{B} \times \nabla \phi}{B^{2}} \\
\dot{v}_{\|} & =0
\end{aligned}
$$

if all $\nabla B$ terms are ignored. For the spatial coordinates of the particle one thus finds

$$
\begin{aligned}
\dot{r} & =\frac{1}{B^{2}}\left(B_{1} \phi_{, z}-\frac{B_{0}}{r} \phi_{, \Theta}\right) \\
\dot{\Theta} & =\frac{v_{\|} B_{1}}{B r}+\frac{B_{0} \phi_{, r}}{B^{2} r} \\
\dot{z} & =\frac{v_{\|} B_{0}}{B}-\frac{B_{1} \phi_{, r}}{B^{2}}
\end{aligned}
$$

Suppose that $B \approx B_{0}$ and introduce the rotational transform as

$$
\iota=\frac{R_{0} B_{1}}{r B_{0}} .
$$

The equations of motion then reduce to (terms that scale with the inverse aspect ratio can be neglected)

$$
\begin{aligned}
\dot{r} & =\frac{r}{R_{0}} l \phi_{, z}-\frac{1}{r B_{0}} \phi_{, \Theta} \cong-\frac{1}{r B_{0}} \phi_{, \Theta} \\
\dot{\Theta} & =\frac{l v_{\|}}{R_{0}}+\frac{1}{r B_{0}} \phi_{, r} \\
\dot{z} & =v_{\|}-\frac{r}{R_{0}} l \phi_{, r} \cong v_{\|} .
\end{aligned}
$$

The $\dot{z}$-equation is easily integrated. When transforming to a new coordinate $p=B_{0} r^{2} / 2$ it can be seen that the remaining system can be cast into Hamiltonian form

$$
\begin{aligned}
\dot{p} & =-\frac{\partial H}{\partial \Theta} \\
\dot{\Theta} & =\frac{\partial H}{\partial p}
\end{aligned}
$$

with $H=v_{\|} \psi / R_{0}+\phi$ and $\partial \psi / \partial p=\iota$. Obviously, the Hamiltonian of the co-moving frame,

$$
\bar{H}=\left(\frac{v_{\|}}{R_{0}} n-\omega\right) p+m H,
$$


is an invariant of the (perturbed) motion. Suppose that the potential varies as $\phi=$ $\phi_{0} \cos \left(m \Theta+n / R_{0} z-\omega t\right)$. Then, a phase

$$
\chi=m \Theta+\frac{n}{R_{0}} z_{0}+\left(\frac{n}{R_{0}} v_{\|}-\omega\right) t
$$

can be defined in the co-moving frame of the wave. The equations of motion in the reference frame of the wave are thus

$$
\begin{aligned}
& \dot{p}=-\frac{\partial \bar{H}}{\partial \chi}=m \phi_{0} \sin \chi \\
& \dot{\chi}=\frac{\partial \bar{H}}{\partial p}=\frac{n}{R_{0}} v_{\|}-\omega+\frac{m}{R_{0}} l v_{\|}+m \cos \chi \frac{\partial \phi_{0}}{\partial p} .
\end{aligned}
$$

Calculating the second time derivative of the phase and Taylor-expanding around $\chi=0$ yields

$$
\begin{aligned}
\ddot{\chi} & =\left[\frac{m}{R_{0}} \frac{\partial \iota}{\partial p} v_{\|}+m \cos \chi \frac{\partial^{2} \phi_{0}}{\partial p^{2}}\right] m \phi_{0} \sin \chi-m \sin \chi \frac{\partial \phi_{0}}{\partial p}\left[\frac{n}{R_{0}} v_{\|}-\omega+\frac{m}{R_{0}} l v_{\|}+m \cos \chi \frac{\partial \phi_{0}}{\partial p}\right] \\
& \cong\left[\frac{m^{2}}{R_{0}} \frac{\partial \iota}{\partial p} v_{\|} \phi_{0}+m^{2} \phi_{0} \frac{\partial^{2} \phi_{0}}{\partial p^{2}}-m \frac{\partial \phi_{0}}{\partial p}\left(v_{\|} k_{\|}-\omega\right)-m^{2}\left(\frac{\partial \phi_{0}}{\partial p}\right)^{2}\right] \chi \\
& \equiv-\omega_{\mathrm{b}}^{2} \chi .
\end{aligned}
$$

Using the resonance condition $v_{\|} k_{\|}=\omega$, the square of the bounce frequency is defined as

$$
\omega_{\mathrm{b}}^{2} \equiv-\left[\frac{m^{2}}{R_{0}} \frac{\partial \iota}{\partial p} v_{\|} \phi_{0}+m^{2} \phi_{0} \frac{\partial^{2} \phi_{0}}{\partial p^{2}}-m^{2}\left(\frac{\partial \phi_{0}}{\partial p}\right)^{2}\right]
$$

and includes terms both linear and quadratic in the field amplitude. This is contrary to conventional Berk-Breizman theory and could hint on the transition from the resonance detuning regime (terms linear in $\phi_{0}$ ) to the radial decoupling regime (quadratic terms). The estimate for $\omega_{\mathrm{b}}$ presented in the main text (see Eq. (31)) is obtained by keeping only the linear term and transforming back to the radial coordinate $r$.

We can use that equation to try to estimate the validity range of the analytical theory [3]. In order to compare with the numerical simulations, we substitute the parameters of the ITPA benchmark and use $v_{\|}=v_{\mathrm{A}} / 3$. The results of this simple estimate for $\omega_{\mathrm{b}}$ are shown in Fig. 12 in blue for the various collision frequencies using the value for $\phi_{0}$ at saturation. Given the value for $\omega_{\mathrm{b}}$ it is then possible to calculate $v_{\text {eff }}$ (red curve in Fig. 12). Two regimes are visible. For $v_{\text {eff }} \ll \gamma_{L}$ the assumptions of Ref. [3] are valid. In this regime the theoretically predicted scaling is confirmed numerically (in the resonance detuning regime). For $v_{\text {eff }} \gg \gamma_{\mathrm{L}}$, on the other hand, the initial assumptions are violated and a new scaling law is found numerically. This simple cylindrical estimate is able to qualitatively predict the transition from one regime to the other. It fits fairly well with our simulation results. 

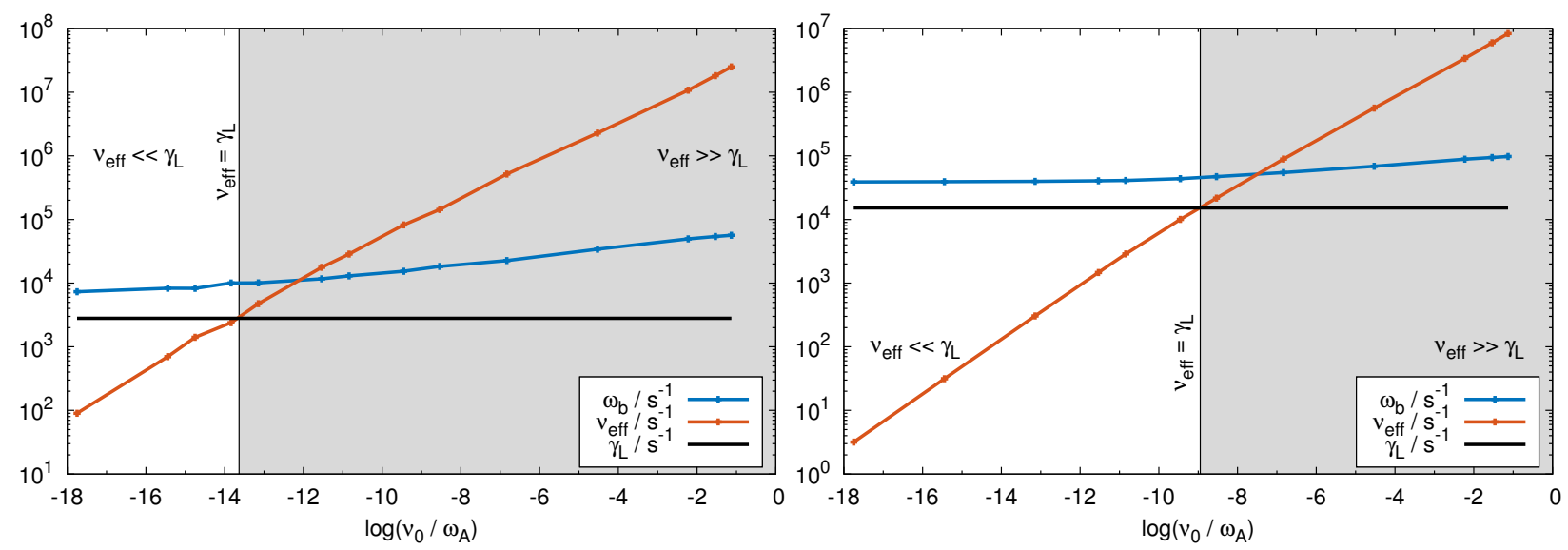

Figure 12: Resulting values of the cylindrical $\omega_{\mathrm{b}}$ estimates (blue) for the low-density (left) and medium-density cases (right). The calculated values for $v_{\text {eff }}$ are shown in red. The primary assumptions of the analytical theory [3] exclude the gray region. 


\section{References}

[1] I. G. Abel, M. Barnes, S. C. Cowley, W. Dorland, and A. A. Schekochihin. Linearized model Fokker-Planck collision operators for gyrokinetic simulations. I. Theory. Physics of Plasmas, 15:122509, 2008.

[2] H. L. Berk and B. N. Breizman. Saturation of a single mode driven by an energetic injected beam. III. Alfvén wave problem. Physics of Fluids B, 2:2246, 1990.

[3] H. L. Berk, B. N. Breizman, and Ye. Huanchun. Scenarios for the nonlinear evolution of alpha-particle-induced Alfvén wave instability. Physical Review Letters, 68:3563, 1992.

[4] S. Briguglio, X. Wang, F. Zonca, G. Vlad, G. Fogaccia, C. Di Troia, and V. Fusco. Analysis of the nonlinear behavior of shear-Alfvén modes in tokamaks based on Hamiltonian mapping techniques. Physics of Plasmas, 21:112301, 2014.

[5] S. Brunner, E. Valeo, and J. A. Krommes. Collisional delta-f scheme with evolving background for transport time scale simulations. Physics of Plasmas, 6:4504, 1999.

[6] V. N. Duarte, H. L. Berk, N. N. Gorelenkov, W. W. Heidbrink, G. J. Kramer, R. Nazikian, D. C. Pace, M. Podestà, B. J. Tobias, and M. A. Van Zeeland. Prediction of nonlinear evolution character of energetic-particle-driven instabilities. Nuclear Fusion, 57:054001, 2017.

[7] H. H. Duong, W. W. Heidbrink, E. J. Strait, T. W. Petrie, R. Lee, R. A. Moyer, and J. G. Watkins. Loss of energetic beam ions during TAE instabilities. Nuclear Fusion, 33:749, 1993.

[8] A. Könies et al. 10th IAEA Technical Meeting on Energetic Particles in Magnetic Confinement Systems (Kloster Seeon), 2007.

[9] S. M. Kaye et al. Progress towards high performance plasmas in the National Spherical Torus Experiment (NSTX). Nuclear Fusion, 45:S168, 2005.

[10] T. B. Fehér. Simulation of the interaction between Alfvén waves and fast particles. PhD thesis, Ernst-Moritz-Arndt-Universität Greifswald, 2013.

[11] G. Y. Fu and J. W. van Dam. Excitation of the toroidicity-induced shear Alfvén eigenmode by fusion alpha particles in an ignited tokamak. Physics of Fluids B, 1:1949, 1989.

[12] P. Helander and D. J. Sigmar. Collisional Transport in Magnetized Plasmas. Cambridge University Press, 2002.

[13] E. Hirvijoki, A. Brizard, A. Snicker, and T. Kurki-Suonio. Monte Carlo implementation of a guiding-center Fokker-Planck kinetic equation. Physics of Plasmas, 20:092505, 2013.

[14] K. Kauffmann. Including Collisions in Gyrokinetic Tokamak and Stellarator Simulations. PhD thesis, Ernst-Moritz-Arndt-Universität Greifswald, 2011.

[15] R. Kleiber, R. Hatzky, A. Könies, A. Mishchenko, and E. Sonnendrücker. An explicit large time step particle-in-cell scheme for nonlinear gyrokinetic simulations in the electromagnetic regime. Physics of Plasmas, 23:032501, 2016.

[16] A. Könies. paper on the model of CKA-EUTERPE, in preparation.

[17] A. Könies, S. Briguglio, N. Gorelenkov, T. Fehér, M. Isaev, Ph. Lauber, A. Mishchenko, D. A. Spong, Y. Todo, W. A. Cooper, R. Hatzky, R. Kleiber, M. Borchardt, G. Vlad, and ITPA EP TG. Benchmark of gyrokinetic, kinetic MHD and gyrofluid codes for the linear calculation of fast particle driven TAE dynamics. 24th IAEA Int. Conf. on Fusion Energy (San Diego, 
CA, 2012), 2012. http://www-naweb.iaea.org/napc/physics/FEC/FEC2012/papers/437_ ITRP134.pdf.

[18] A. Könies and D. Eremin. Coupling of Alfvén and sound waves in stellarator plasmas. Physics of Plasmas, 17:012107, 2010.

[19] V. Kornilov, R. Kleiber, R. Hatzky, L. Villard, and G. Jost. Gyrokinetic global threedimensional simulations of linear ion-temperature-gradient modes in Wendelstein 7-X. Physics of Plasmas, 11:3196, 2004.

[20] J. Lang, G. Fu, and Y. Chen. Nonlinear simulation of toroidal Alfvén eigenmode with source and sink. Physics of Plasmas, 17:042309, 2010.

[21] M. Lesur, Y. Idomura, K. Shinohara, X. Garbet, and the JT-60 Team. Spectroscopic determination of kinetic parameters for frequency sweeping Alfvén eigenmodes. Physics of Plasmas, 17:122311, 2010.

[22] A. V. Melnikov, L. G. Eliseev, E. Ascasíbar, A. Cappa, F. Castejón, C. Hidalgo, T. Ido, J. A. Jiménez, A. S. Kozachek, L. I. Krupnik, M. Liniers, S. E. Lysenko, K. Nagaoka, J. L. de Pablos, A. Shimizu, S. E. Sharapov, M. V. Ufimtsev, S. Yamamoto, HIBP Group, and TJ-II team. Transition from chirping to steady NBI-driven Alfvén modes caused by magnetic configuration variations in the TJ-II stellarator. Nuclear Fusion, 56:076001, 2016.

[23] A. V. Melnikov, L. G. Eliseev, F. Castejón, P. O. Khabanov, A. S. Kozachek, L. I. Krupnik, M. Liniers, S. E. Lysenko, , J. L. de Pablos, S. E. Sharapov, M. V. Ufimtsev, V. N. Zenin, HIBP Group, and TJ-II team. Study of NBI-driven chirping mode properties and radial location by the heavy ion beam probe in the TJ-II stellarator. Nuclear Fusion, 56:112019, 2016.

[24] T. Sunn Pedersen, M. Otte, S. Lazerson, P. Helander, S. Bozhenkov, C. Biedermann, T. Klinger, R. C. Wolf, H.-S. Bosch, and The Wendelstein 7-X Team. Confirmation of the topology of the Wendelstein 7-X magnetic field to better than 1:100,00. Nature Communications, 7:13493, 2016.

[25] M. N. Rosenbluth and P. H. Rutherford. Excitation of Alfvén Waves by High-Energy Ions in a Tokamak. Physical Review Letters, 34:1428, 1975.

[26] S. Satake, R. Kanno, and H. Sugama. Development of a Non-Local Neoclassical Transport Code for Helical Configurations. Plasma and Fusion Research, 3:S1062, 2008.

[27] C. Slaby, R. Kleiber, and A. Könies. Combining electromagnetic gyro-kinetic particle-in-cell simulations with collisions. Computer Physics Communications, 218:1, 2017.

[28] Christoph Slaby, Axel Könies, and Ralf Kleiber. Numerical investigation of non-perturbative kinetic effects of energetic particles on toroidicity-induced Alfvén eigenmodes in tokamaks and stellarators. Physics of Plasmas, 23:092501, 2016.

[29] T. Takizuka and H. Abe. A binary collision model for plasma simulation with a particle code. Journal of Computational Physics, 25:205, 1977.

[30] Y. Todo, R. Seki, D. A. Spong, H. Wang, Y. Suzuki, S. Yamamoto, N. Nakajima, and M. Osakabe. Comprehensive magnetohydrodynamic hybrid simulations of fast ion driven instabilities in a Large Helical Device experiment. Physics of Plasmas, 24:081203, 2017.

[31] T. Vernay. Numerical study of electron-ion collision effects on trapped electron modes with the gyrokinetic code ORB5. Master's thesis, École Polytechnique Fédérale de Lausanne, 2008 .

[32] T. Vernay, S. Brunner, L. Villard, B. F. McMillan, S. Jolliet, T. M. Tran, A. Bottino, and J. P. Graves. Neoclassical equilibria as starting point for global gyrokinetic microturbulence 
simulations. Physics of Plasmas, 17:122301, 2010.

[33] X. Wang, S. Briguglio, Ph. Lauber, V. Fusco, and F. Zonca. Structure of wave-particle resonances and Alfvén mode saturation. Physics of Plasmas, 23:012514, 2016.

[34] Zhixuan Wang, Zhihong Lin, Ihor Holod, W. .W. Heidbrink, B. Tobias, Michael Van Zeeland, and M. .E. Austin. Radial Localization of Toroidicity-Induced Alfvén Eigenmodes. Physical Review Letters, 111:145003, 2013.

[35] R. B. White, E. Fredrickson, D. Darrow, M. Zarnstorff, R. Wilson, S. Zweben, K. Hill, Yang Chen, and Guoyong Fu. Toroidal Alfvén eigenmode-induced ripple trapping. Physics of Plasmas, 2:2871, 1995.

[36] H. S. Zhang, Z. Lin, W. Deng, I. Holod, Z. X. Wang, Y. Xiao, and W. L. Zhang. Nonlinear dynamics of beta-induced Alfvén eigenmodes in tokamak. Physics of Plasmas, 20:012510, 2013.

[37] M. Zhou and R. White. Collisional dependence of Alfvén mode saturation in tokamaks. Plasma Physics and Controlled Fusion, 58:125006, 2016. 